\title{
Use of high potency statins and rates of admission for acute kidney injury: multicenter, retrospective observational analysis of administrative databases
}

\author{
(c) (1) (8)
}

Colin R Dormuth assistant professor ${ }^{1}$, Brenda R Hemmelgarn associate professor ${ }^{2}$, J Michael Paterson scientist ${ }^{3}$, Matthew T James assistant professor ${ }^{2}$, Gary F Teare director of quality management and analysis ${ }^{4}$, Colette B Raymond research scientist ${ }^{5}$, Jean-Philippe Lafrance assistant professor $^{6}$, Adrian Levy head ${ }^{7}$, Amit X Garg professor of medicine ${ }^{8}$, Pierre Ernst professor of medicine $^{9}$, Canadian Network for Observational Drug Effect Studies (CNODES)

${ }^{1}$ Department of Anesthesiology, Pharmacology and Therapeutics, University of British Columbia, Vancouver, Victoria, BC, Canada V8W 1Y2; ${ }^{2}$ Department of Medicine, University of Calgary, Calgary, Canada; ${ }^{3}$ Institute for Clinical Evaluative Sciences, Toronto, Canada; ${ }^{4} \mathrm{Health}$ Quality Council, Saskatoon, Canada; ${ }^{5}$ Manitoba Centre for Health Policy, University of Manitoba, Winnipeg, Canada; ${ }^{6}$ Department of Medicine, University of Montreal, Montreal, Canada; ${ }^{7}$ Community Health and Epidemiology, Dalhousie University, Halifax, Canada; ${ }^{8}$ Division of Nephrology, University of Western Ontario, London, Canada; ${ }^{9}$ McGill University, Centre for Clinical Epidemiology, Lady Davis Institute, Jewish General Hospital, Montreal, Canada

\begin{abstract}
Objective To quantify an association between acute kidney injury and use of high potency statins versus low potency statins.

Design Retrospective observational analysis of administrative databases, using nine population based cohort studies and meta-analysis. We performed as treated analyses in each database with a nested case-control design. Rate ratios for different durations of current and past statin exposure to high potency or low potency statins were estimated using conditional logistic regression. Ratios were adjusted for confounding by high dimensional propensity scores. Meta-analytic methods estimated overall effects across participating sites.

Setting Seven Canadian provinces and two databases in the United Kingdom and the United States.

Participants 2067639 patients aged 40 years or older and newly treated with statins between 1 January 1997 and 30 April 2008. Each person hospitalized for acute kidney injury was matched with ten controls.

Intervention A dispensing event was new if no cholesterol lowering drug or niacin prescription was dispensed in the previous year. High potency statin treatment was defined as $\geq 10 \mathrm{mg}$ rosuvastatin, $\geq 20 \mathrm{mg}$ atorvastatin, and $\geq 40 \mathrm{mg}$ simvastatin; all other statin treatments were defined as low potency. Statin potency groups were further divided into cohorts with or without chronic kidney disease.
\end{abstract}

Main outcome measure Relative hospitalization rates for acute kidney injury.

Results Of more than two million statin users (2 008003 with non-chronic kidney disease; 59636 with chronic kidney disease), patients with similar propensity scores were comparable on measured characteristics. Within 120 days of current treatment, there were 4691 hospitalizations for acute kidney injury in patients with non-chronic kidney injury, and 1896 hospitalizations in those with chronic kidney injury. In patients with non-chronic kidney disease, current users of high potency statins were $34 \%$ more likely to be hospitalized with acute kidney injury within 120 days after starting treatment (fixed effect rate ratio $1.34,95 \%$ confidence interval 1.25 to 1.43). Users of high potency statins with chronic kidney disease did not have as large an increase in admission rate $(1.10,0.99$ to 1.23$) \cdot x^{2}$ tests for heterogeneity confirmed that the observed association was robust across participating sites.

Conclusions Use of high potency statins is associated with an increased rate of diagnosis for acute kidney injury in hospital admissions compared with low potency statins. The effect seems to be strongest in the first 120 days after initiation of statin treatment.

\section{Introduction}

Evidence has indicated that statin use could lead to unintended adverse renal effects. ${ }^{1-3}$ A large scale, randomized controlled 
trial-JUPITER (Justification for the Use of Statins in Prevention: an Intervention Trial Evaluating Rosuvastatin)—compared high dose (20 mg) rosuvastatin with placebo in almost 18000 patients. ${ }^{4}$ Data subsequently reported to the United States Food and Drug Administration (FDA) showed a non-significant increase of $19 \%$ in acute renal failure (risk ratio $1.19,95 \%$ confidence interval 0.61 to 2.31$).^{5}$ The non-significant risk increased further to $35 \%(1.35,0.81$ to 2.23$)$ when the endpoint also included doubling of serum creatinine. A large, population based cohort study of over two million patients also reported that statin use was associated with a greater than $50 \%$ increase in risk of acute renal failure, with evidence of raised risk within the first year of statin use, and a dose-response effect. ${ }^{3}$ In a Canadian product monograph for rosuvastatin, the most potent statin, the manufacturer also reported renal impairment in some patients who received $80 \mathrm{mg}$ of the drug in investigational clinical trials. ${ }^{6}$

It remains unclear whether statin therapy is specifically associated with greater adverse renal effects. In the light of data alluding to a possible dose response, we aimed to explore a possible association between statins and kidney injury by comparing patients who were prescribed high potency statins with those prescribed low potency statins. The main advantage of using exposure to lower potency statins as a reference group was an expectation that a substantial amount of unmeasured confounding by indication would thus be eliminated. A comparison of high versus low potency might also be informative for treatment where statin use is clearly supported by evidence of total mortality benefit (for example, in patients who have had myocardial infarction), but where evidence of incremental benefit for high potency statins versus low potency statins is questionable. ${ }^{7}$

In the inaugural study of the Canadian Network for Observational Drug Effect Studies (CNODES), ${ }^{8}$ we used the administrative healthcare records of two million people to assess the association between treatment with high versus low potency statins and hospitalization for acute kidney injury in patients with and without chronic kidney disease. CNODES is part of the new Canadian Drug Safety and Effectiveness Network, initiated by Health Canada and administered by the Canadian Institutes of Health Research. The principal aim of CNODES is to collaboratively analyze existing healthcare databases using observational research methods, to provide rapid answers to questions on drug safety and effectiveness.

\section{Methods}

\section{Setting and source population}

We used a common analytical protocol to conduct studies of statins in acute kidney injury in seven Canadian provinces (Alberta, British Columbia, Manitoba, Nova Scotia, Ontario, Quebec, and Saskatchewan; source population 33 million in 2011), as well as in two international databases in the United Kingdom (General Practice Research Database (GPRD), 11.6 million) and the US (Caremark-Medicare database, 1.2 million). The study populations were patients aged 40 years or older, newly treated with statins between 1 January 1997 and 30 April 2008. In three provinces, only patients aged 65 years and older were available, and four of the databases were established after 1997.

\section{Data sources}

All participating jurisdictions contributed data from their respective administrative healthcare databases. Prescription data included, at a minimum, patient level records specifying the drug dispensed, the quantity or days' supply of treatment, and the date of dispensation. The GPRD was an exception in that written prescriptions were recorded rather than actual drugs dispensed. Physician claims and hospitalizations databases included date of service encounter or hospital admission, up to 25 ICD-9 or ICD-10 (international classification of disease, 9th or 10th revisions) diagnosis codes, and procedure and billing codes. The Saskatchewan databases and GPRD included laboratory test data. The GPRD also included body mass index and smoking status. All databases have been used extensively in observational research.

\section{Cohorts of patients according to statin use}

Our analytical approach was to conduct as treated analyses using nested case-control methodology. This design is a good alternative to cohort analysis when studying time dependent exposures, and its computational efficiency is well suited to analyzing rare events in large databases. ${ }^{9}$ In each jurisdiction, patients from the source population were included in cohorts of chronic or non-chronic kidney disease if they were newly dispensed a statin between 1 January 1997 (or one year after the beginning of data availability) and 30 April 2008.

We defined a dispensing event as new if no cholesterol lowering drug (World Health Organization Anatomical Therapeutic Class $\mathrm{C} 10)$ or prescription for niacin was dispensed in the previous 365 days. The date of the new statin prescription was defined as the cohort entry date. Daily statin doses were categorized according to their potency in lowering serum concentrations of low density lipoprotein (LDL) cholesterol.

High potency statin treatment was defined as at least $10 \mathrm{mg}$ rosuvastatin, at least $20 \mathrm{mg}$ atorvastatin, and at least $40 \mathrm{mg}$ simvastatin; all other statin treatments were defined as having low potency. Our categorization was derived from a systematic review and meta-analysis of randomized controlled trials that quantified the effects of statins on LDL cholesterol concentration. ${ }^{10}$ The analysis showed that statins clustered around three levels of LDL reduction. One group (defined as low potency) produced a reduction of about $35 \%$, another (defined as high potency) reduced concentrations by about $45 \%$; $80 \mathrm{mg}$ rosuvastatin lowered concentrations by $60 \%$. This classification is similar to ones used elsewhere. ${ }^{11-13}$ We grouped $80 \mathrm{mg}$ rosuvastatin with the middle group, owing to relatively few of such prescriptions. Therefore, statins were categorized as high or low in our study, according to whether they would produce a theoretical reduction in LDL cholesterol concentration of less than $45 \%$, or at least $45 \% .^{12}$

Patients were excluded if they were younger than 40 years $(<66$ years in jurisdictions that only had drug data for senior citizens), had less than one year of enrolment in their medical system, or received any cholesterol lowering drug or underwent dialysis in the previous year. Finally, we assigned eligible patients to cohorts of chronic and non-chronic kidney disease according to whether they had at least one hospitalization or physician medical encounter for non-dialysis dependent, chronic kidney disease in the previous three years. The web appendix lists definitions of chronic kidney disease.

\section{Acute kidney injury endpoint}

The primary endpoint was hospitalization for acute kidney injury, defined using a validated algorithm (diagnosis code for acute kidney injury in any of the following listed diagnoses: ICD-9 584, 584.5, 584.6, 584.7, 584.8, or 584.9; ICD-10 N17, N17.0, N17.1, N17.2, N17.8, or N17.9). ${ }^{14}$ 


\section{High dimensional propensity scores}

High dimensional propensity scores (hdPS) were estimated for all patients in the cohorts. The hdPS algorithm is available as downloadable SAS software files from the Brigham and Women's Hospital. ${ }^{16}$ As described in detail elsewhere, ${ }^{17}$ hdPS is an empirically driven, multistep process to adjust for confounding bias in observational studies. The hdPS algorithm prioritizes thousands of variables at the drug, diagnostic, procedural, and demographic level according to their potential to cause multiplicative bias in the estimate of an exposure-outcome association (for example, rate ratio). Typically, the 500 variables most likely to cause bias are included in a propensity score model. We used logistic regression to estimate the predicted probability (as measured by the propensity score) of exposure to high potency statins versus low potency statins, conditional on all of the included covariates.

In addition to the 500 covariates empirically selected for the propensity score models, we also included the following prespecified covariates: year of cohort entry, hospitalization, order for a laboratory test, greater than four distinct drugs dispensed, greater than four physician visits, hypertensive disease, hypercholesterolemia, peripheral vascular disease, congestive heart failure, and injury or poisoning, all within the year prior to cohort entry (web appendix). Propensity score models were estimated using logistic regression.

\section{As treated analysis}

In each cohort described above, we defined a follow-up end date for each patient as the earliest occurrence of an acute kidney injury endpoint, date of death, date of emigration, 24 months after initiation on statin treatment, a dispensing for cerivastatin, or 31 March 2010. Cerivastatin was not among the statins included in the analysis. We identified all cases of the endpoint occurring within patients' follow-up windows and defined the date of admission for acute kidney injury as the index date. For each case, we randomly selected ten controls from a matched risk set comprising patients of the same sex and age within one year (if no controls were available, within five years), who also entered the cohort within 90 days before or after the case's cohort entry date.

We used conditional logistic regression to estimate matched odds ratios for three mutually exclusive durations of current exposure ( $\leq 120,121-365$, and 366-730 days) and past exposure (no exposure within 120 days of the index date). Patients who received both a high and low potency statin in the same current exposure category or as past exposures were categorized as receiving high potency statins in that particular category. Adjusted matched odds ratios were estimated by including the tenth of the propensity score (nine binary indicator variables); diagnosis of diabetes; and prior use of angiotensin II converting enzyme inhibitors or receptor blockers, non-steroidal

anti-inflammatory drugs, thiazide diuretics, and loop diuretics. In addition, we assessed potential drug-drug interactions with statins by including main effect variables and interaction variables for three types of compounds: macrolide antibiotics, fibrates, and calcium channel blockers.

\section{Fixed cohort analysis}

We also conducted fixed cohort analyses on the cohorts in each jurisdiction, where the statin exposure category was defined by the initial prescription at cohort entry. We expected that fixed cohort estimates would be biased towards the null hypothesis, mostly because of dilution of risk due to treatment non-adherence and dose escalation. The hdPS model used in this analysis was the same as the model in the as treated analysis, but it estimated effects for 180 days of follow-up after cohort entry instead of two years. For patients with non-chronic or chronic kidney disease, we used logistic regression to estimate odds ratios for statin potency and acute kidney injury. Odds ratio estimates were reported as relative risks, and the logistic regressions included tenth of propensity score (nine binary indicator variables); category of age (in five year groups); diagnosis of diabetes; and prior use of angiotensin II converting enzyme inhibitors or receptor blockers, non-steroidal anti-inflammatory drugs, thiazide diuretics, and loop diuretics.

\section{Meta-analysis}

All analyses were undertaken independently at each site, which were kept blind to results from the other sites until the meta-analysis was conducted. For the meta-analysis, we pooled the studies from each site using either fixed effect or random effects models according to results of $\chi^{2}$ tests for heterogeneity. ${ }^{18}$ Matched odds ratios were reported as rate ratios. Inverse variance weighted odds ratios and $95 \%$ confidence intervals were calculated to estimate the total effect across all study populations.

\section{Results}

\section{Study populations}

Overall, 2067639 patients in participating databases were newly exposed to statins during the study period, of which 673410 $(33 \%)$ received high potency statins (table $\downarrow$ ); the sizes of the source populations varied broadly. We recorded 59636 patients with chronic kidney injury using statins within three years before cohort entry. The table also shows baseline characteristics of the overall study population, matched and unmatched on propensity score, which demonstrates the comparability of patients using high potency and low potency statins on measured factors conditional on their propensity scores. The mean age of study participants was 68 years, although in jurisdictions with younger patients the mean age was between 61 and 65 years. Fifty percent of patients were women.

\section{Rates of acute kidney injury}

In the Canadian jurisdictions with patients younger than 65 years, the risk of hospitalization for acute kidney injury at six months in patients with non-chronic kidney disease receiving low dose statins (that is, the reference group) ranged from 1.2-1.4 per 1000 patients (in British Columbia, Manitoba, and Saskatchewan) to 3.5 per 1000 patients (in Quebec). The corresponding risk was 1.0 per 1000 patients in the GPRD. In the remaining jurisdictions with data for adults aged 65 years and older only, the corresponding risk was 3.1 per 1000 patients thousand in Ontario, Nova Scotia, and Alberta, and 4.0 per 1000 patients in the US Medicare Caremark database. Rates were substantially higher in patients with a history of chronic kidney disease: 23-45 per 1000 patients in first six months after statin initiation in Canada, 10 per 1000 patients in the GPRD, and 63 per 1000 patients in the US Medicare Caremark database.

\section{As treated analysis}

Patients who started high potency statins were $34 \%$ more likely to be hospitalized for acute kidney injury than those who started low potency statins in the first 120 days of treatment (fixed effect rate ratio $1.34,95 \%$ confidence interval 1.25 to 1.43 ; fig $1 \Downarrow)$. The elevated effect was attenuated with longer durations 
of current exposure. The rate of hospitalization for acute kidney injury did not increase significantly in patients with chronic kidney disease $(1.10,0.99$ to 1.23 ; fig $2 \Downarrow)$.

The $\chi^{2}$ tests for heterogeneity were not significant for all meta-analyses except for past use in patients with chronic kidney disease. In that analysis, a random effects estimate of the total effect was reported. We checked for effect modification of statin potency by macrolide antibiotics, calcium channel blockers, and fibrates, but did not find any significant interactions. The fixed cohort analysis also showed a significantly increased risk for acute kidney injury within six months of treatment initiation in both groups of patients with non-chronic and chronic kidney disease (web fig).

\section{Discussion}

In this study of over two million patients newly treated with a statin, we found a significant relative increase of $34 \%$ in the rate of hospitalization for acute kidney injury within 120 days of initiation for patients receiving high potency statins versus low potency statins. Our as treated analysis was based on an expectation that less than perfect treatment persistence and a high tendency for subsequent switching to higher doses might cause a fixed cohort analysis to underestimate the risk while actually on treatment. Loss of some patients to follow-up and competing risks also could have contributed to an underestimation of the effect. The as treated analysis, which allowed quantification of risk from different durations of current exposure, indicated that risk remained elevated for at least two years.

Although we used multiplicative models to answer our study questions, it is the subsequent translation of relative risks into numbers needed to treat to harm that provides the most useful metric for prescribers, regulators, and decision makers. In this regard, we estimate that 1700 patients with non-chronic kidney disease need to be treated with a high potency statin instead of a low potency statin for 120 days to cause one additional hospitalization for acute kidney injury. A number of 1700 patients is sufficiently large for there not to be enough patients enrolled in randomized trials to find an association between acute kidney injury and statin use with high precision. However, our definition of acute kidney injury was chosen to be highly specific and thus probably excluded a number of patients with real but milder cases, which could have underestimated the absolute risk.

Further studies are necessary to determine the biological mechanism linking statins to kidney injury. The elevated risk in patients using high potency statins could be related to an increased risk of rhabdomyolysis. Another mechanism could be the statin induced suppression of coenzyme Q10, a fat soluble enzyme with antioxidant properties. Statins have been shown to block the production of coenzyme Q10, ${ }^{19}{ }^{20}$ and placebo controlled trials of coenzyme Q10 treatment in humans and animals with kidney disease have shown improvements in renal function within 28 days of use. ${ }^{20}{ }^{21}$ Other studies have shown an association between statin treatment and proteinuria. ${ }^{52}$ Pleiotrophic statin effects should also be contemplated.

\section{Comparison with existing evidence}

According to data from the JUPITER trial, as published by the FDA, ${ }^{5}$ the maximum likelihood estimate of the relative risk was 1.11 for any renal event over a median follow-up duration of 1.9 years, and 1.19 for acute renal failure. Our rate ratio estimate was 1.15 for acute kidney injury for patients treated for one to two years. Over all treatment durations in our analysis, the average rate ratio was 1.17 , or 1.20 if past treatment was excluded. The compatibility of these results with our hypothesis, their agreement with the maximum likelihood estimate observed in the large JUPITER trial, and the high degree of precision obtained from our study population, together lend meaningful support for this increased risk.

Similarity between our results and other epidemiologic studies is mixed. One multicenter study of statin use and acute kidney injury in patients with community acquired pneumonia (CAP) reported an odds ratio of 1.32 for acute kidney injury in patients with CAP who received statins compared with statin naive patients. ${ }^{23}$ By contrast, a meta-analysis of four observational studies of rosuvastatin, designed to study multiple outcomes but which included renal failure, reported no difference between rosuvatstain and other statins. ${ }^{24}$ This meta-analysis is impossible to interpret from a statin potency perspective, because the control patients received other statins of all different potencies.

It is also important to note that most statin treatment in randomized trials was of low potency. The JUPITER trial showed that about 450 patients needed to be treated with $20 \mathrm{mg}$ rosuvastatin per day instead of placebo for two years to prevent one death from myocardial infarction, stroke, or cardiovascular disease (combined endpoint). ${ }^{4}$ It remains to be shown whether the number needed to treat to benefit with high potency statins instead of low potency statins (versus placebo) would outweigh the combined risk of acute kidney injury, rhabdomyolysis, and diabetes.

\section{Implications}

In a meta-analysis of clinical trials of statins, the Cholesterol Treatment Trialists (CTT) reported that more intensive statin treatment in secondary prevention was associated with a $0.3 \%$ reduction in absolute risk in major coronary events per year of treatment, compared with less intensive statin treatment over a mean of five years. ${ }^{25}$ Some have argued that an absolute risk reduction of $0.3 \%$ per year overstates what could be expected in typical clinical practice. ${ }^{7}$ Importantly, the trials comparing more intensive treatment with less intensive treatment included in the CTT meta-analysis were mostly comparisons of a low dose of a particular statin versus the highest possible dose of that same statin. In reality, clinicians would not choose between, for example, $10 \mathrm{mg}$ of atorvastatin and $80 \mathrm{mg}$ of atorvastatin. Given what is likely to be a small magnitude of incremental cardiovascular benefit of high potency statins over low potency statins in reality, a pressing question is how to identify patients for whom the risk-benefit balance for high potency statin treatment is unfavourable.

\section{Methodological considerations}

Chronic kidney disease status could have been misclassified at cohort entry using ICD codes from administrative data. However, in view of the specificity of these definitions (99\%), ${ }^{26}$ we do not believe that any potential misclassification (that is, in relation to the small number of participants with chronic kidney disease being misclassified as having non-chronic kidney disease) would be substantial enough to have influenced the estimate of effect in the patients with non-chronic kidney disease.

Our outcome of acute kidney injury was defined using a validated algorithm with high specificity but low sensitivity. Thus, the absolute rates of acute kidney injury identified in hospitalization records would underestimate the true incidence based on changes in serum creatinine. However, we do not expect that our definition of acute kidney injury would have 
resulted in differential misclassification of patients during the study period. Acute kidney injury would have been an unexpected outcome of treatment, and patients with true injuries misclassified as non-injuries would have been those not hospitalized for the disease, and were probably less clinically relevant than those captured by our specified definition.

Although our results are much more compatible with the maximum likelihood estimates of renal harm in the JUPITER trial than with a null hypothesis, confounding by indication remains a possible threat to the validity of our results. To minimize this bias, the reference group consisted of patients who also received statin treatment. We adjusted for a broad spectrum of possible confounders using hdPS scores. There was probably a non-significant trend over time towards increasing use of high potency statins, which necessitated the inclusion of calendar year of cohort entry as a covariate in the models.

There was also a small imbalance after propensity score matching between patients using high potency and low potency statins in the proportion that had congestive heart failure (table), which is a known independent risk factor for acute kidney injury. In view of the difference between and the relative numbers of patients exposed to high potency statins versus low potency statins, we estimate that at least a 25 -fold independent relative increase in the risk of acute kidney injury associated with congestive heart failure would be needed to spuriously produce our risk estimate of 1.34 in our population (that is, association between acute kidney injury with statin potency). A 2.0 risk of acute kidney injury associated with congestive heart failure would produce a spurious association between statin potency and acute kidney injury of about 1.01. Diagnosis of congestive heart failure was included in the propensity score models, and outcome models were adjusted for use of loop diuretics in the year before cohort entry.

This study was made possible through data sharing agreements between CNODES member research centres and the respective provincial governments of Alberta, British Columbia, Manitoba, Nova Scotia, Ontario, Quebec and Saskatchewan. We thank Sebastian Schneeweiss and Jun Liu of the Division of Pharmacoepidemiology and Pharmacoeconomics at Brigham and Women's Hospital for their expertise and assistance in analyzing the US Medicare data and use of the high-dimensional propensity score method; and James M Wright for sharing expert knowledge of pharmacology and clinical trial evidence for statins.

CNODES investigators: Samy Suissa (principal investigator, McGill University); David Henry and Michael Paterson (Ontario); Colin Dormuth (British Columbia); Brenda Hemmelgarn (Alberta); Gary Teare (Saskatchewan); Patricia Martens and Patricia Caetano (Manitoba); Pierre Ernst, Jacques LeLorier, and Robert Platt (Québec); and Adrian Levy (Nova Scotia).

Contributors: CRD was responsible for overseeing the development of the study protocol and the creation of a data analysis plan that was adapted for use in each jurisdiction and each data set. All variables and all outcomes were specified in that protocol and any deviations from this were for technical reasons only (for example, variable coding of administrative data in certain jurisdictions). Except where prevented by insufficient data or small cell size limitations, all prespecified outcomes in the protocol are reported. A copy of the study protocol is available on request. All coauthors contributed to roundtable discussions on protocol development and all provided critical revisions to the manuscript. $\mathrm{BRH}, \mathrm{MTJ}, \mathrm{J}-\mathrm{PL}$, and AXG were instrumental in defining the acute kidney injury outcome and identifying patients with chronic kidney disease. CBR assisted with exposure definitions. JMP, GFT, AL, and $P E$ contributed to numerous study design issues and critical revision of the manuscript. CRD was responsible for drafting the manuscript and incorporating suggestions of coauthors and he is the guarantor of the manuscript. The final manuscript was approved by the publications subcommittee of CNODES.

Funding: CNODES is funded by a grant from Health Canada, the Drug Safety and Effectiveness Network, and the Canadian Institutes for Health Research that oversaw the peer review and funding process.

Competing interests: All authors have completed the ICMJE uniform disclosure form at www.icmje.org/coi_disclosure.pdf and declare: support from Health Canada, the Drug Safety and Effectiveness Network, and the Canadian Institutes for Health Research for the submitted work; PE receives consultancy and speaker's fees from Astra Zeneca, Boehringer Ingelheim, Merck, and Nycomed, and a research grant from Boehringer Ingelheim; AL receives consultancy fees from Oxford Outcomes (now Icon), a company that carries out contracted work for pharmaceutical companies; no other relationships or activities that could appear to have influenced the submitted work.

Ethical approval: Ethics approval for each study was obtained from the respective academic institutions in each province.

Data sharing: CNODES is not permitted to release individual level data or aggregated data with small cell sizes. The analytical protocol for this analysis is available on request.

1 Wolfe SM. Dangers of rosuvastatin identified before and after FDA approval. Lancet 2004:363:2189-90.

2 Kiortsis DN, Filippatos TD, Mikhailidis DP, Elisaf MS, Liberopoulos EN. Statin-associated adverse effects beyond muscle and liver toxicity. Atherosclerosis 2007;195:7-16.

3 Hippisley-Cox J, Coupland C. Unintended effects of statins in men and women in England and Wales: population based cohort study using the QResearch database. BMJ 2010;340:c2197.

4 Ridker PM, Danielson E, Fonseca FA, Genest J, Gotto AM Jr, Kastelein JJ, et al; JUPITER study group. Rosuvastatin to prevent vascular events in men and women with elevated C-reactive protein. N Engl J Med 2008;359:2195-207.

5 Roberts MD. CRESTOR (Rosuvastatin calcium) NDA 21-366 JUPITER. United States Food and Drug Administration Endocrinologic and Metabolic Drugs Advisory Committee. 2009. www.fda.gov/downloads/advisorycommittees/committeesmeetingmaterials/drugs/ endocrinologicandmetabolicdrugsadvisorycommittee/ucm194918.pdf.

6 Canadian Pharmacists Association. Compendium of pharmaceuticals and specialties. The Association, 2004.

7 Sniderman A, Thanassoulis G, Couture P, Williams K, Alam A, Furberg CD. Is lower and lower better and better? A re-evaluation of the evidence from the Cholesterol Treatment Trialists' Collaboration meta-analysis for low-density lipoprotein lowering. J Clin Lipidol 2012;6:303-9.

8 Suissa S, Henry D, Caetano P, Dormuth CR, Ernst P, Hemmelgarn B, et al; for the CNODES investigators. CNODES: the Canadian Network for Observational Drug Effect Studies. Open Med 2012;6:E134-40.

9 Essebag V, Platt RW, Abrahamowicz M, Pilote L. Comparison of nested case-control and survival analysis methodologies for analysis of time-dependent exposure. BMC Med Res Methodol 2005:5:5.

10 Law MR, Wald NJ, Rudnicka AR. Quantifying effect of statins on low density lipoprotein cholesterol, ischaemic heart disease, and stroke: systematic review and meta-analysis. BMJ 2003;326:1423.

11 Kheterpal S, Tremper KK, Englesbe MJ, O'Reilly M, Shanks AM, Fetterman DM, et al. Predictors of postoperative acute renal failure after noncardiac surgery in patients with previously normal renal function. Anesthesiology 2007;107:892-902.

12 Ouattara A, Benhaoua H, Le Manach Y, Mabrouk-Zerguini N, Itani O, Osman A, et al. Perioperative statin therapy is associated with a significant and dose-dependent reduction of adverse cardiovascular outcomes after coronary artery bypass graft surgery. $J$ Cardiothorac Vasc Anesth 2009;23:633-8.

13 Molnar AO, Coca SG, Devereaux PJ, Jain AK, Kitchlu A, Luo J, et al. Statin use associates with a lower incidence of acute kidney injury after major elective surgery. J Am Soc Nephrol 2011;22:939-46.

14 Waikar SS, Wald R, Chertow GM, Curhan GC, Winkelmayer WC, Liangos O, et al. Validity of International Classification of Diseases, ninth revision, clinical modification codes for acute renal failure. J Am Soc Nephrol 2006;17:1688-94

15 Hwang YJ, Shariff SZ, Gandhi S, Wald R, Clark E, Fleet JL, et al. Validity of the International Classification of Diseases, tenth revision code for acute kidney injury in elderly patients at presentation to the emergency department and at hospital admission. BMJ Open 2012;2.

16 High-dimensional Propensity Score (hd-PS), version 1. 2010. www.hdpharmacoepi.org/ download/.

17 Schneeweiss S, Rassen JA, Glynn RJ, Avorn J, Mogun H, Brookhart MA. High-dimensional propensity score adjustment in studies of treatment effects using health care claims data. Epidemiology 2009;20:512-22.

18 DerSimonian R, Laird N. Meta-analysis in clinical trials. Control Clin Trials 1986;7:177-88

19 McMurray JJ, Dunselman P, Wedel H, Cleland JG, Lindberg M, Hjalmarson A, et al; CORONA Study Group. Coenzyme Q10, rosuvastatin, and clinical endpoints in heart failure: a pre-specified sub study of CORONA (controlled rosuvastatin multinational study in heart failure). J Am Coll Cardiol 2010;56:1196-204

20 Singh RB, Khanna HK, Niaz MA. Randomized, double-blind placebo-controlled trial of coenzyme Q10 in chronic renal failure: discovery of a new role. J Nutr Environ Med 2000;10:281-8.

21 Ishikawa A, Kawarazaki H, Ando K, Fujita M, Fujita T, Homma Y. Renal preservation effect of ubiquinol, the reduced form of coenzyme Q10. Clin Exp Nephrol 2011;15:30-3. 


\section{What is already known on this topic}

Various data sources have pointed to a possible harmful effect of statins on the kidney, although previous analyses have not specifically looked at acute kidney injury

\section{What this study adds}

Prescription of high potency statins ( $\geq 10 \mathrm{mg}$ rosuvastatin, $\geq 20 \mathrm{mg}$ atorvastatin, $\geq 40 \mathrm{mg}$ simvastatin) is associated with an increased rate of hospital admission for acute kidney injury, compared with lower potency statins

Increased risk of admission occurs early after starting statin treatment and remains elevated for at least two years

Prescribers should consider this potential risk when contemplating use of high potency statins in clinical practice, particularly when treatment with a low potency statin is an option

Alsheikh-Ali AA, Ambrose MS, Kuvin JT, Karas RH. The safety of rosuvastatin as used in common clinical practice: a postmarketing analysis. Circulation 2005;111:3051-7.

23 Murugan R, Weissfeld L, Yende S, Singbartl K, Angus DC, Kellum JA; Genetic and Inflammatory Markers of Sepsis (GenIMS) Investigators. Association of statin use with risk and outcome of acute kidney injury in community-acquired pneumonia. Clin J Am Soc Nephrol 2012;7:895-905.

24 García Rodríguez LA, Herings R, Johansson S. Use of multiple international healthcare databases for the detection of rare drug-associated outcomes: a pharmacoepidemiological programme comparing rosuvastatin with other marketed statins. Pharmacoepidemiol Drug Saf 2010;19:1218-24.

25 Cholesterol Treatment Trialists' (CTT) Collaborators. Efficacy and safety of more intensive lowering of LDL cholesterol: a meta-analysis of data from 170000 participants in 26 randomised trials. Lancet 2010;376:1670-81.
26 Vlasschaert ME, Bejaimal SA, Hackam DG, Quinn R, Cuerden MS, Oliver MJ, et al. Validity of administrative database coding for kidney disease: a systematic review. Am J Kidney Dis 2011;57:29-43.

Accepted: 30 January 2013

\section{Cite this as: BMJ 2013;346:f880}

This is an open-access article distributed under the terms of the Creative Commons Attribution Non-commercial License, which permits use, distribution, and reproduction in any medium, provided the original work is properly cited, the use is non commercial and is otherwise in compliance with the license. See: http://creativecommons.org/licenses/by$\mathrm{nc} / 2.0 /$ and http://creativecommons.org/licenses/by-nc/2.0/legalcode. 


\section{Table}

\begin{tabular}{|c|c|c|c|c|c|c|c|c|}
\hline \multicolumn{9}{|c|}{$\begin{array}{r}\text { Table 1| Baseline characteristics of patients receiving high potency a } \\
\text { Patients without chronic kidney disease }\end{array}$} \\
\hline & \multicolumn{2}{|c|}{$\begin{array}{c}\text { Subset with matched } \\
\text { propensity score }\end{array}$} & \multicolumn{2}{|c|}{ Full cohort } & \multicolumn{2}{|c|}{$\begin{array}{l}\text { Subset with matched } \\
\text { propensity score* }\end{array}$} & \multicolumn{2}{|c|}{ Full cohort } \\
\hline & $\begin{array}{l}\text { High potency } \\
\text { statins }(n=633 \\
054)\end{array}$ & $\begin{array}{l}\text { Low potency } \\
\text { statins }(n=633 \\
054)\end{array}$ & $\begin{array}{l}\text { High potency } \\
\text { statins } \\
(\mathrm{n}=651069)\end{array}$ & $\begin{array}{l}\text { Low potency } \\
\text { statins }(n=1 \\
356934)\end{array}$ & $\begin{array}{l}\text { High potency } \\
\text { statins }(n=20 \\
511)\end{array}$ & $\begin{array}{l}\text { Low potency } \\
\text { statins }(\mathrm{n}=20 \\
\quad 511)\end{array}$ & $\begin{array}{c}\text { High } \\
\text { potency } \\
\text { statins }(n=22 \\
341)\end{array}$ & $\begin{array}{l}\text { Low potency } \\
\text { statins }(n=37 \\
\text { 295) }\end{array}$ \\
\hline \multicolumn{9}{|l|}{ Age (years) } \\
\hline $40-49$ & $53063(8.4)$ & $53043(8.4)$ & $54588(8.4)$ & $98040(7.2)$ & $479(2.3)$ & $477(2.3)$ & $521(2.3)$ & $1172(3.1)$ \\
\hline $50-59$ & $103608(16.4)$ & 102999 (16.3) & $105892(16.3)$ & 205985 (15.2) & $1118(5.4)$ & $1116(5.4)$ & $1220(5.5)$ & $2249(6.0)$ \\
\hline $60-64$ & $62598(9.9)$ & $62663(9.9)$ & $63753(9.8)$ & $131137(9.7)$ & $911(4.4)$ & $921(4.5)$ & $994(4.4)$ & $1813(4.9)$ \\
\hline $65-69$ & $133890(21.2)$ & $134037(21.2)$ & 137811 (21.2) & 287547 (21.2) & $2783(13.6)$ & $2782(13.6)$ & $3099(13.9)$ & $5487(14.7)$ \\
\hline $70-74$ & $119624(18.9)$ & $120076(19.0)$ & $123221(18.9)$ & 279905 (20.6) & $3986(19.4)$ & 3990 (19.5) & $4337(19.4)$ & $7797(20.9)$ \\
\hline $75-79$ & $87110(13.8)$ & $87113(13.8)$ & $89903(13.8)$ & $201711(14.9)$ & $4493(21.9)$ & $4472(21.8)$ & $4820(21.6)$ & $8189(22.0)$ \\
\hline$\geq 80+$ & 73161 (11.6) & $73123(11.6)$ & 75901 (11.7) & 152609 (11.2) & $6741(32.9)$ & $6753(32.9)$ & $7350(32.9)$ & $10588(28.4)$ \\
\hline \multicolumn{9}{|l|}{ Sex } \\
\hline Women & $291877(46.1)$ & $291861(46.1)$ & 302883 (46.5) & $706318(52.1)$ & $8827(43.0)$ & $8814(43.0)$ & $9741(43.6)$ & $17230(46.2)$ \\
\hline Men & 341177 (53.9) & 341193 (53.9) & $348186(53.5)$ & $650616(47.9)$ & $11684(57.0)$ & $11697(57.0)$ & $12600(56.4)$ & 20065 (53.8) \\
\hline \multicolumn{9}{|l|}{ Diagnoses } \\
\hline Hypertensive disease & $245372(38.8)$ & 239908 (37.9) & 255282 (39.2) & 536985 (39.6) & $13442(65.5)$ & $13029(63.5)$ & $14137(63.3)$ & 22437 (60.2) \\
\hline Hypercholesterolemia & $170979(27.0)$ & 178730 (28.2) & 177377 (27.2) & $438124(32.3)$ & $4700(22.9)$ & $4619(22.5)$ & $4865(21.8)$ & $8373(22.5)$ \\
\hline $\begin{array}{l}\text { Peripheral vascular } \\
\text { disease }\end{array}$ & $15144(2.4)$ & 4250 (2.3) & $740(2.4)$ & 33528 (2.5) & $1385(6.8)$ & $1328(6.5)$ & $1768(7.9)$ & 2939 (7.9) \\
\hline $\begin{array}{l}\text { Congestive heart } \\
\text { failure }\end{array}$ & $38036(6.0)$ & $29107(4.6)$ & 40317 (6.2) & $61699(4.5)$ & $5938(28.9)$ & $4834(23.6)$ & $6984(31.3)$ & $9073(24.3)$ \\
\hline Diabetes & $134289(21.2)$ & $139806(22.1)$ & $138702(21.3)$ & $296000(21.8)$ & $7116(34.7)$ & $7044(34.3)$ & $7797(34.9)$ & $13041(35.0)$ \\
\hline Injury or poisoning & $72122(11.4)$ & $68889(10.9)$ & 76574 (11.8) & $171879(12.7)$ & $2807(13.7)$ & $2489(12.1)$ & $2875(12.9)$ & $4242(11.4)$ \\
\hline \multicolumn{9}{|l|}{ No of hospitalizations } \\
\hline None & $418648(66.1)$ & $453553(71.6)$ & 459485 (70.6) & $\begin{array}{c}1057112 \\
(77.9)\end{array}$ & 7559 (36.9) & $9512(46.4)$ & $7950(35.6)$ & $18084(48.5)$ \\
\hline 1 & 85508 (13.5) & $71387(11.3)$ & $103286(15.9)$ & $170078(12.5)$ & $6135(29.9)$ & $5411(26.4)$ & $6683(29.9)$ & $9126(24.5)$ \\
\hline 2 & 36995 (5.8) & $27415(4.3)$ & $44882(6.9)$ & $64843(4.8)$ & $3204(15.6)$ & $2694(13.1)$ & $3620(16.2)$ & $4590(12.3)$ \\
\hline$\geq 3$ & $38481(6.1)$ & 27199 (4.3) & $43599(6.7)$ & $64867(4.8)$ & $3613(17.6)$ & $2896(14.1)$ & $4087(18.3)$ & $5496(14.7)$ \\
\hline \multicolumn{9}{|l|}{ Drugs } \\
\hline $\begin{array}{l}\text { Prescription } \\
\text { acetaminophen }\end{array}$ & $64042(10.1)$ & $61515(9.7)$ & $65545(10.1)$ & $148468(10.9)$ & $5070(24.7)$ & $4784(23.3)$ & $5268(23.6)$ & $8420(22.6)$ \\
\hline Prescription NSAID & $147029(23.2)$ & $144000(22.7)$ & $150810(23.2)$ & 328350 (24.2) & $4940(24.1)$ & 4701 (22.9) & $5370(24.0)$ & $8799(23.6)$ \\
\hline ACE inhibitor & $236534(37.4)$ & 214661 (33.9) & $242816(37.3)$ & $436912(32.2)$ & $12013(58.6)$ & $11444(55.8)$ & $12950(58.0)$ & 20602 (55.2) \\
\hline $\begin{array}{l}\text { Angiotensin II receptor } \\
\text { blocker }\end{array}$ & $84527(13.4)$ & 80889 (12.8) & 88255 (13.6) & $135863(10.0)$ & $4996(24.4)$ & $4958(24.2)$ & $5622(25.2)$ & $8036(21.5)$ \\
\hline Thiazide diuretics & $110216(17.4)$ & 112030 (17.7) & $113738(17.5)$ & 240556 (17.7) & $5738(28.0)$ & 5795 (28.3) & $6234(27.9)$ & $9749(26.1)$ \\
\hline Loop diuretics & 53896 (8.5) & 45664 (7.2) & 55777 (8.6) & $102710(7.6)$ & $8073(39.4)$ & 7347 (35.8) & 8748 (39.2) & $13712(36.8)$ \\
\hline $\begin{array}{l}\text { Potassium sparing } \\
\text { diuretics }\end{array}$ & $37514(5.9)$ & 35419 (5.6) & $39717(6.1)$ & $87843(6.5)$ & $2895(14.1)$ & 2699 (13.2) & $3462(15.5)$ & $5160(13.8)$ \\
\hline$\beta$ blockers & $195998(31.0)$ & $164369(26.0)$ & $201952(31.0)$ & 360234 (26.5) & $11105(54.1)$ & $9738(47.5)$ & 11912 (53.3) & 16964 (45.5) \\
\hline $\begin{array}{l}\text { Calcium channel } \\
\text { blockers }\end{array}$ & $135197(21.4)$ & $131628(20.8)$ & $138906(21.3)$ & 311416 (22.9) & $10108(49.3)$ & 10098 (49.2) & $10814(48.4)$ & 18572 (49.8) \\
\hline Antibiotics & 220881 (34.9) & 218761 (34.6) & 227209 (34.9) & $511004(37.7)$ & $10508(51.2)$ & $10366(50.5)$ & $11390(51.0)$ & $19317(51.8)$ \\
\hline
\end{tabular}

Data are no (\%) of patients. NSAID=non-steroidal anti-inflammatory drug; ACE=angiotensin-converting-enzyme. 
(continued)

\begin{tabular}{ccccc}
\multicolumn{3}{c}{ Patients without chronic kidney disease } \\
\hline \multicolumn{2}{c}{$\begin{array}{c}\text { Subset with matched } \\
\text { propensity score* }\end{array}$} & & Full cohort \\
\cline { 1 - 1 } High potency & Low potency & & High potency & Low potency \\
statins $(n=633$ & statins $(n=633$ & & statins & statins $(n=1$ \\
$054)$ & $054)$ & & $(n=651069)$ & $356934)$
\end{tabular}

\begin{tabular}{|c|c|c|}
\hline \multicolumn{3}{|c|}{ Patients with chronic kidney disease } \\
\hline $\begin{array}{l}\text { Subset with matched } \\
\text { propensity score* }\end{array}$ & Full c & cohort \\
\hline $\begin{array}{l}\text { High potency Low potency } \\
\text { statins }(n=20 \text { statins }(n=20\end{array}$ & $\begin{array}{c}\text { High } \\
\text { potency } \\
\text { statins }(n=22\end{array}$ & $\begin{array}{l}\text { Low potency } \\
\text { statins }(n=37\end{array}$ \\
\hline 511) & 341) & 295) \\
\hline
\end{tabular}

*One to one matching was used. Within the same sex and age within one year, each patient using high potency statins was matched to a patient using low potency statin with the nearest propensity score. If multiple controls had the same propensity score, one was chosen at random. 


\section{Figures}

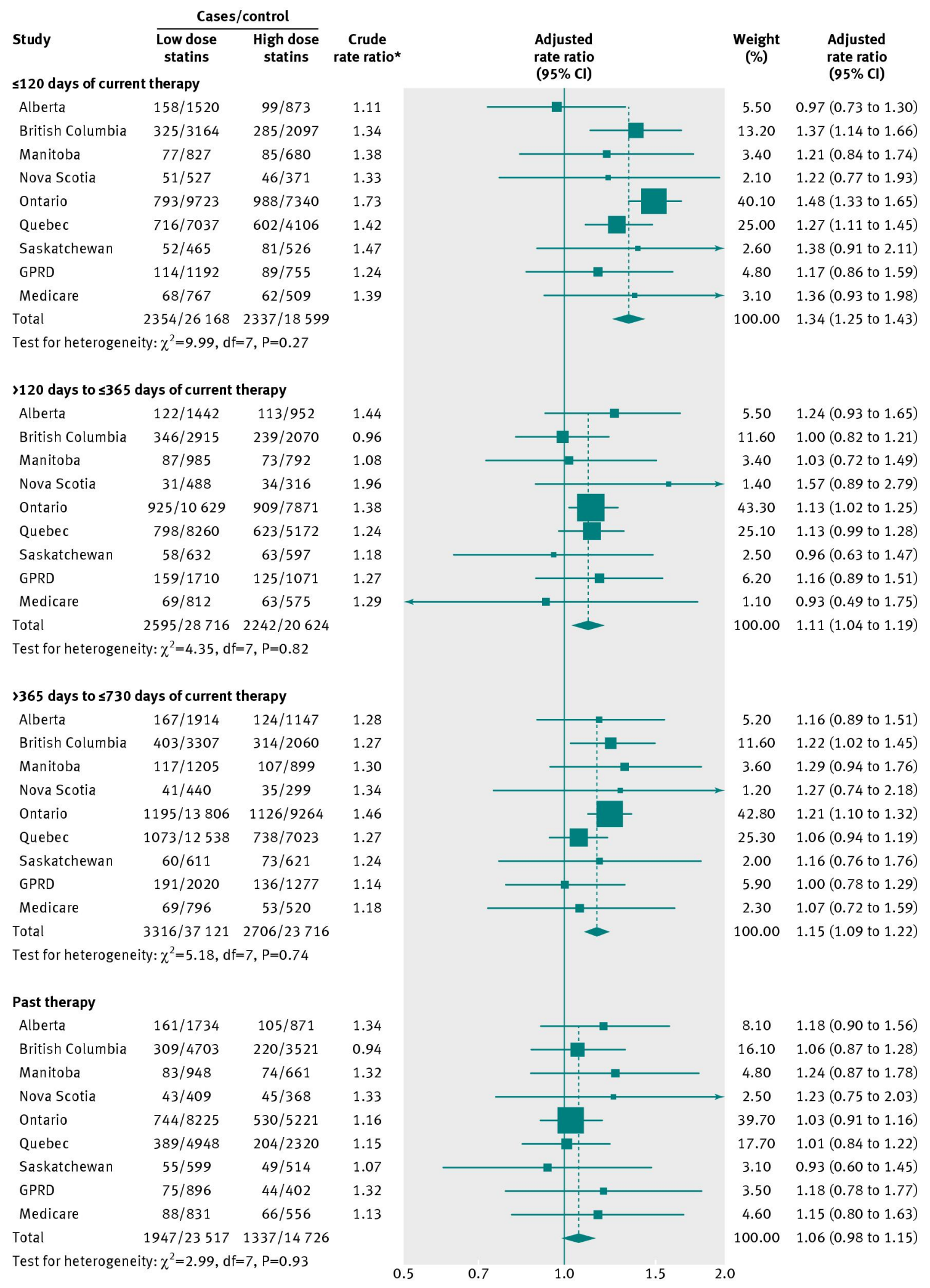

Fig 1 As treated analysis of hospitalization for acute kidney injury up to two years after cohort entry in patients without chronic kidney disease. ${ }^{*}$ Crude rate ratios were obtained from conditional logistic regression, conditioned on the matching variables age, sex, and cohort entry date 


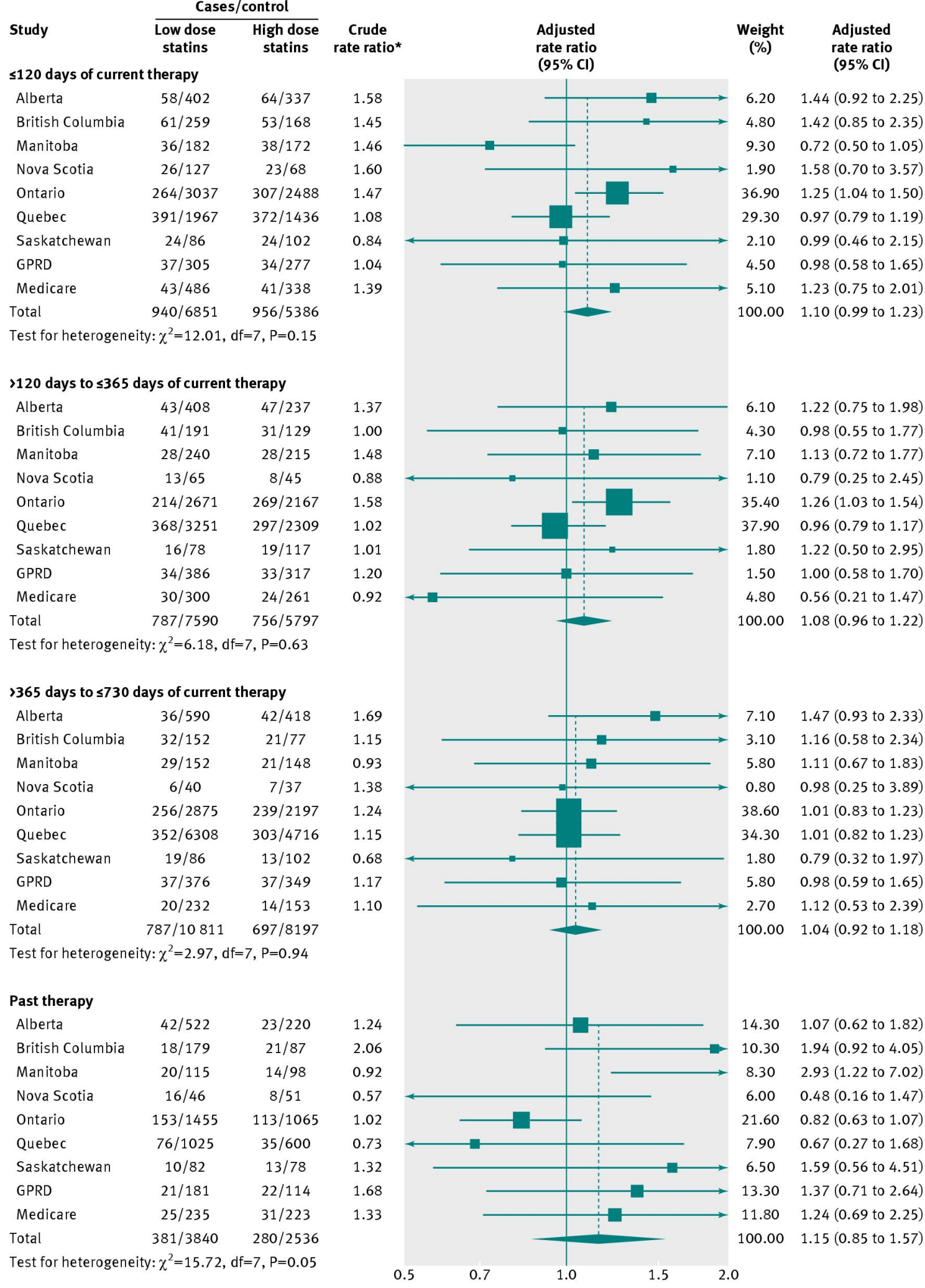

Fig 2 As treated analysis of hospitalization for acute kidney injury up to two years after cohort entry in patients with chronic kidney disease. ${ }^{*}$ Crude rate ratios were obtained from conditional logistic regression, conditioned on the matching variables age, sex, and cohort entry date 